\title{
Predictive value of serum proinflammatory biomarkers in respiratory infections in children and clinical-therapeutic correlations
}

\author{
Titiana Cornelia Cotoi ${ }^{1}$, Raluca Niculescü ${ }^{2}$, Adrian-Horatiu Sabau², Lorena Melit ${ }^{3}$, \\ Oana Cristina Marginean ${ }^{3}$, Adina Hutanu $u^{4,5}$, Daniela Lucia Muntean ${ }^{6}$ \\ ${ }^{1}$ IOSUD, "George Emil Palade" University of Medicine, Pharmacy, Science and Technology, \\ Tg. Mures, Romania \\ ${ }^{2}$ Department of Pathology, Mureș County Clinical Hospital, Tg. Mures, Romania \\ ${ }^{3}$ Pediatrics Clinic, "George Emil Palade" University of Medicine, Pharmacy, Science and Technology, \\ Tg. Mures, Romania \\ ${ }^{4}$ Department of Laboratory Medicine, "George Emil Palade" University of Medicine, Pharmacy, Science \\ and Technology, Tg. Mures, Romania \\ ${ }^{5}$ CCAMF, "George Emil Palade" University of Medicine, Pharmacy, Science and Technology, \\ Tg. Mures, Romania \\ ${ }^{6}$ Faculty of Pharmacy, "George Emil Palade" University of Medicine, Pharmacy, Science and Technology, \\ Tg. Mures, Romania
}

\begin{abstract}
Introduction. Pneumonia in children causes a high rate of morbidity and mortality, especially in young children. The evolution and prognosis are influenced by a series of clinical-biological parameters, in relation to the child's age and the therapy received.

Objectives. Assessment of proinflammatory status in patients with pneumonia.

Material and methods. The study was conducted at the Pediatrics Clinic I within the Emergency County Clinical Hospital in Tg. Mures, Romania, between September 2018 and December 2019 and included 95 patients.

Results. Analysis of the serum level of C-reactive protein, the high-sensitive form (hsCRP), showed a positive correlation, statistically significant between its level at admission and the number of neutrophils at admission $(p=03169$, $r=0.2801$ ), respectively a negative correlation, statistically significant between its level at discharge and the level of leukocytes $(p=0.0253, r=-0.2911)$, monocytes $(p=0.0014, r=-0.4105)$ and neutrophils $(p=0.0335, r=-0.2793)$ at discharge. From the three monitored cytokines (IL-1 $\beta$, IL- 6 and TNF- $\alpha$ ) statistically significant correlations were observed for TNF- $\alpha$. Thus, a positive, statistically significant correlation was identified between lymphocyte values ( $p$ $=0.0007, r=0.3448)$, monocytes $(p=0.00563, r=0.2896)$ and platelets $(p=0.0335, r=0.2195)$ at admission with the values of the serum marker TNF- $\alpha$. Also, a negative, statistically significant correlation was identified between hemoglobin values $(p=0.0034, r=-0.328)$ and hematocrit $(p=0.0004, r=-0.3932)$ at discharge and the TNF- $a$ serum marker values. The following correlations were observed: negative correlation, statistically significant between TNF- $\alpha$ and NLR $(p=0.0144, r=-0.2601)$, NPR $(p=0.0473, r=-0.2051)$ and PLR $(p=0,0138, r=-0.2602)$, positive correlation, statistically significant between hsCRP at admission and NLR $(p=0.0124, r=0.3264)$, NPR $(p=0.0073$, $r=0.3460)$ and positive correlation, statistically significant between hsCRP at discharge and NLR $(p=0.0189, r=$ $0.3128)$, PLR ( $p=0.0036, r=0.3831)$.

Conclusions. Our study showed that the values of neutrophils, lymphocytes, monocytes and platelets, NLR, PLR and TNF- $\alpha$ were significantly increased in pneumonia, correlating with C-reactive protein, highlighting the early inflammatory status in this condition.
\end{abstract}

Keywords: pneumonia in children, cytokines, IL-1 $\beta$, IL-6, TNF- $\alpha$, antibiotic therapy

Corresponding author:

Pharm. Titiana Cornelia Cotoi

E-mail: titianacotoi@yahoo.com
Article History:

Received: 25 May 2020

Accepted: 12 June 2020 


\section{INTRODUCTION}

Pneumonia in children leads to a high rate of morbidity and mortality, especially in young children (1). The evolution and prognosis are influenced by a series of clinical-biological parameters, in relation to the child's age: newborn, infant, toddler, preadolescent. Severe cases with poor therapeutic response are often followed by patient death (2).

There are several risk factors for severe respiratory infections: prematurity, low birth weight (LBW) or very low birth weight (VLBW), patients with age under 3 months, and patients with congenital cardiac malformations (left-to-right cardiac shunt) or lung malformations or other chronic pathologies (heart failure, chronic obstructive pulmonary disease, diabetes etc.) $(3,4)$. Other significant risk factors are lack of breastfeeding, malnutrition, poor access to health services and low socioeconomic status of the child's family $(5,6)$.

The etiology of pneumonia in children varies greatly with age and only partially overlaps with what happens in the adult population (7). Thus, from newborns to the age of 3 weeks, bacterial infections with Streptococcus group B, some Gram-negative germs and Streptococcus pneumoniae predominate. After the age of 3 weeks, until the first three months of life, viral infections with respiratory syncytial virus, rhinoviruses, parainfluenza virus, influenza virus, adenovirus predominate, to which are added bacterial infections with Streptococcus pneumoniae, H. influenzae. After the age of 4 months, up to 4 years, in addition to the ones mention above, there are also Mycoplasma pneumoniae, Streptococcus group A. After the age of 5, viral infections with Mycoplasma pneumoniae, Streptococcus pneumoniae, Chlamydophila pneumoniae, $H$. influenzae predominate, and viral infections are rarer, being represented by influenza virus, adenovirus and other respiratory viruses (8-10).

Along with the usual laboratory tests, more and more studies report the role of biomarkers in identifying inflammatory status - inflammatory cytokines - interleukin-1 (IL-1), interleukin-6 (IL-6), interleukin-12 (IL-12) and tumor necrosis factor- $\alpha(\mathrm{TNF}-\alpha)$ and oxidative stress (catalase, total antioxidant capacity, superoxide desmutase, oxidized glutathione/glutathione reduced ratio) $(11,12)$.

\section{PURPOSE}

The aim of the study is to assess the proinflammatory status in children with antibiotic-treated pneumonia.

\section{MATERIAL AND METHODS}

The study was a prospective one conducted at the Pediatrics Clinic I within the Emergency County Clinical Hospital in Tg. Mures, Romania, between September 2018 and December 2019, and included 95 patients, aged between one month and 16 years. The inclusion criteria in the study were: children with pneumonia, hospitalized in the Pediatric Clinic, where informed consent was obtained from parents or legal guardians. Exclusion criteria included: other infectious or non-infectious pathologies of other organ systems and lack of cooperation from relatives.

The analyzed variables were: patient age, patient sex, patient weight, hospitalization diagnosis, complete blood count at hospital admission and discharge, reactive protein $\mathrm{C}$ values at hospital admission and discharge, serum levels of IL- $1 \beta$, IL- 6 and TNF- $\alpha$, antibiotic therapy, the evolution of the patient and the number of days of hospitalization.

In order to determine the serum level of cytokines, the multiplex technique and xMAP technology were used, on the Flexmap 3D analyzer using the Human Cytokine Magnetic bead panel Milliplex kit, according to the instructions given by the manufacturer. Performance characteristics of the kit: detection limit of $1.2 \mathrm{pg} / \mathrm{ml}$ for TNF- $\alpha, 1.0 \mathrm{pg} / \mathrm{ml}$ for IL- $1 \beta$ and $1.3 \mathrm{pg} /$ $\mathrm{ml}$ for IL-6, and coefficient of variation intra- and inter-run below $5 \%$ respectively $15 \%$.

Descriptive and analytical statistics were performed using SPSS v17.0, GraphPad Prism 8 and XLSTAT-Lite 2015 software for Windows 10 Pro. In order to eliminate the aberrant values, the Grubbs test was performed. The Kolmogorov-Smirnov test was applied before the correlation tests were applied. In order to correlate the values $\mathrm{n}$ the blood count and the serum levels of IL-1 $\beta$, IL- 6 and TNF- $\alpha$, the Pearson test for parametric data and the Spearman test for nonparametric data were performed. The significance threshold chosen for $\mathrm{p}$ was 0.05 .

All parents or relatives have signed informed consent for the inclusion of their children in the study. The study was conducted according to the principles of the Helsinki Declaration and was approved by the Ethics Commission of the "George Emil Palade" University of Medicine, Pharmacy, Science and Technology in Tg. Mures no. 71/27.04.2018.

\section{RESULTS}

The average age of the children in our study was $31.19+/-33.75$ months (with a range between one month and 195 months (16 years and 3 months). In our study, most cases of respiratory infections were 


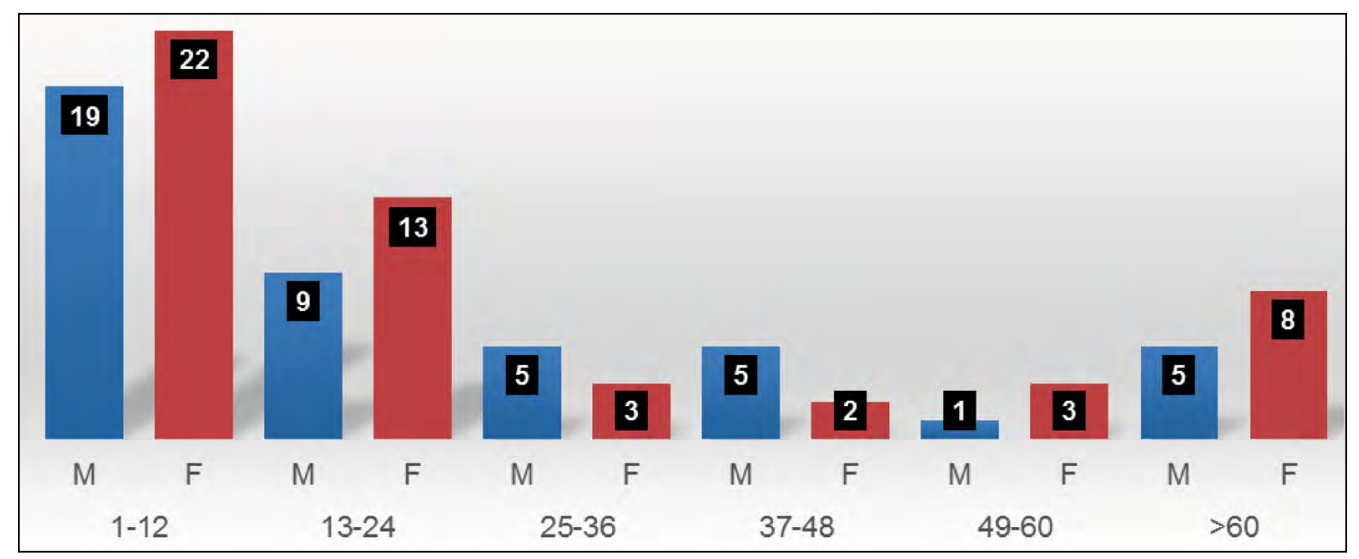

FIGURE 1. Distribution by age groups and by sex of patients

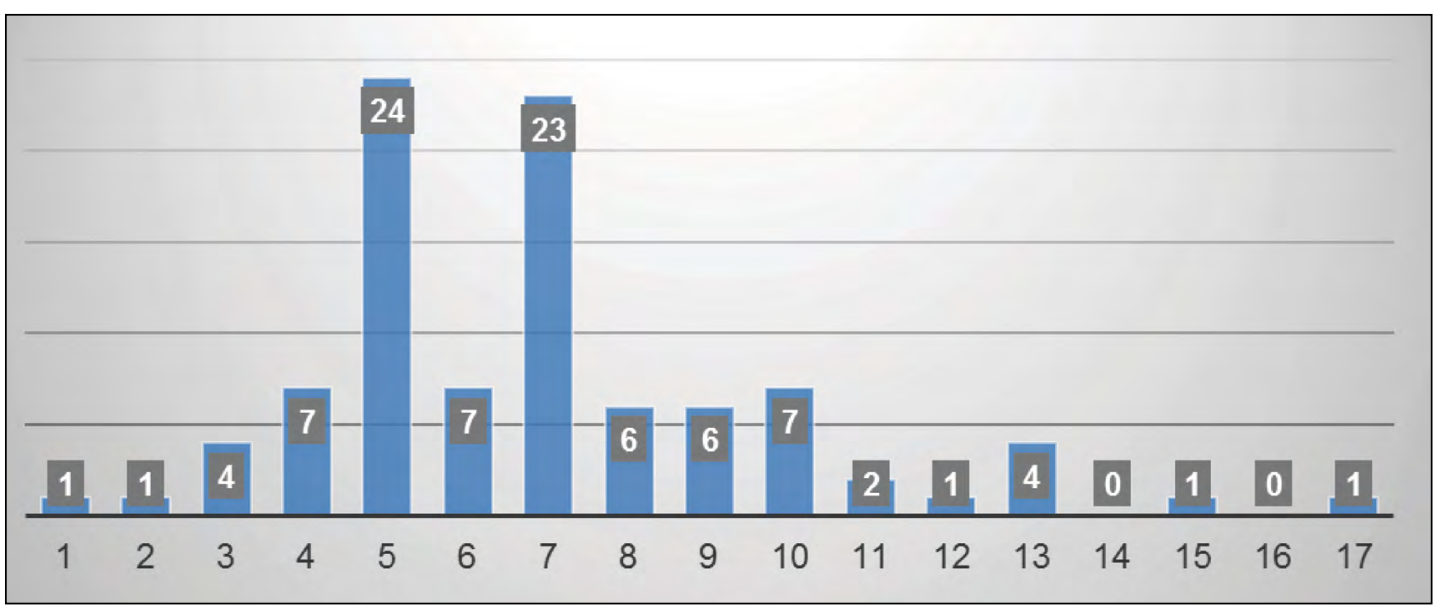

FIGURE 2. Distribution of cases according to the number of days of hospitalization

recorded) in the first two years of life: 63 cases $(66.31 \%)$, of which in the first year of life 41 cases $(43.15 \%)$ and 22 cases $(23.15 \%)$ in the second year of life. Among the risk factors present in patients from the target group we mention: malnutrition (12 cases $-12.63 \%)$, anemia (42 cases $-44.21 \%)$, social cases (11 cases $-11.57 \%)$, congenital heart malformations with left-right shunt (two cases $-2.10 \%$ ) and epilepsy with chronic encephalopathy (one case $-1.05 \%$ ). The distribution by sex shows a slight predominance of females in 51 cases $(53.68 \% \%)$, compared to male patients in 44 cases $(46.31 \%)$. The average weight of the patients was $11.4+/-7.52 \mathrm{~kg}$, with a minimum of $3.5 \mathrm{~kg}$ and a maximum of $47 \mathrm{~kg}$. The distribution by sex and age groups of the cases studied is illustrated in figure 1.

An important parameter followed was the number of hospitalization days, which ranged from one-day hospitalization to 15 or 17 days in severe cases, with an average of 6.91 days (standard deviation SD +/2.82) days for all study group. Of the 95 patients included in the study, 88 patients had a favorable evolu- tion, and patients with multiple comorbidities were discharged in an improved condition. The graphical representation according to the number of hospitalization days is presented in figure 2 .

The proposed treatments for patients with pneumonia in our group included the administration of antibiotics in 68 patients $(71.27 \%)$. From the total of 68 patients who underwent antibiotic treatment, $63 \mathrm{had}$ a favorable evolution. In 5 patients there was no improvement in symptoms under initial treatment and they needed to add another antibiotic or replace the initial treatment with a new treatment regimen. Most of the patients received Ceftriaxone (27 patients $39.70 \%$ of those treated with antibiotics). Ampicillin was given to 17 patients $(25 \%$ of those treated with antibiotics). Other antibiotics administered were: Clindamycin (16 patients - 23.52\%), Cefuroxime (6 patients $-8.82 \%)$, Amoxicillin with clavulanic acid (one patient $-1.47 \%$ ) and Clarithromycin (one patient $-1.47 \%$ ). All patients treated with Cefuroxime or Ampicillin, either alone or in combination with another antibiotic, had a favorable evolution. 
TABLE 1. Correlations between hematological parameters and inflammatory markers

\begin{tabular}{|c|c|c|c|c|c|c|c|c|c|}
\hline \multirow{2}{*}{\multicolumn{2}{|c|}{ The analyzed variables }} & \multicolumn{2}{|c|}{$\mathrm{IL}-\mathbf{1} \boldsymbol{\beta} \mathrm{pg} / \mathrm{ml}$} & \multicolumn{2}{|c|}{ IL-6 pg/ml } & \multicolumn{2}{|c|}{ TNF $\alpha \mathrm{pg} / \mathrm{ml}$} & \multicolumn{2}{|c|}{ PCR mg/dl } \\
\hline & & $n$ & $p$ & $n$ & $p$ & $n$ & $p$ & $n$ & $p$ \\
\hline \multirow[b]{2}{*}{$\begin{array}{l}\mathrm{Hb} \\
\mathrm{g} / \mathrm{dl}\end{array}$} & Admission & 67 & 0.5668 & 66 & 0.4178 & 94 & 0.1594 & 61 & 0.3527 \\
\hline & Discharge & 59 & 0.1461 & 55 & 0.7432 & 78 & $\begin{array}{c}\mathbf{0 . 0 0 3 4} \\
r=-0.328\end{array}$ & 59 & 0.3240 \\
\hline \multirow[b]{2}{*}{$\mathrm{Htc} \%$} & Admission & 66 & 0.6429 & 66 & 0.4999 & 94 & 0.0724 & 61 & 0.2768 \\
\hline & Discharge & 58 & 0.2371 & 55 & 0.5196 & 78 & $\begin{array}{c}\mathbf{0 . 0 0 0 4} \\
r=-0.393\end{array}$ & 59 & 0.1292 \\
\hline \multirow{2}{*}{$\begin{array}{l}\text { Le } \\
10^{3} / \mu l\end{array}$} & Admission & 65 & 0.8799 & 64 & 0.3419 & 92 & 0.1914 & 61 & 0.8109 \\
\hline & Discharge & 58 & 0.5174 & 55 & 0.4596 & 78 & 0.6411 & 59 & $\begin{array}{c}\mathbf{0 . 0 2 5 3} \\
r=-0.2911\end{array}$ \\
\hline \multirow{2}{*}{$\begin{array}{c}\mathrm{Li} \\
10^{3} / \mu \mathrm{l}\end{array}$} & Admission & 66 & 0.9194 & 66 & 0.7565 & 93 & $\begin{array}{c}\mathbf{0 . 0 0 0 7} \\
r=0.3448\end{array}$ & 60 & 0.0605 \\
\hline & Discharge & 56 & 0.3478 & 53 & 0.4631 & 76 & 0.0626 & 58 & 0.3896 \\
\hline \multirow{2}{*}{$\begin{array}{c}\text { Mo } \\
10^{3} / \mu l\end{array}$} & Admission & 64 & 0.8380 & 63 & 0.1069 & 90 & $\begin{array}{c}\mathbf{0 . 0 0 5 6} \\
r=0.2896\end{array}$ & 60 & 0.9506 \\
\hline & Discharge & 57 & 0.2267 & 54 & 0.3788 & 77 & 0.0990 & 58 & $\begin{array}{c}\mathbf{0 . 0 0 1 4} \\
r=-0.4105\end{array}$ \\
\hline \multirow{2}{*}{$\begin{array}{c}\mathrm{Ne} \\
10^{3} / \mu l\end{array}$} & Admission & 66 & 0.5759 & 63 & 0.4649 & 90 & 0.2198 & 61 & $\begin{array}{c}\mathbf{0 . 0 3 1 7} \\
r=0.2801\end{array}$ \\
\hline & Discharge & 56 & 0.8610 & 53 & 0.3050 & 76 & 0.1883 & 57 & $\begin{array}{c}\mathbf{0 . 0 3 5 4} \\
r=-0.2793\end{array}$ \\
\hline \multirow{2}{*}{$\begin{array}{c}P \\
10^{3} / \mu l\end{array}$} & Admission & 67 & 0.8598 & 66 & 0.9630 & 94 & $\begin{array}{c}0.0335 \\
r=0.2195\end{array}$ & 59 & 0.0636 \\
\hline & Discharge & 57 & 0.1529 & 54 & 0.3232 & 76 & 0.0723 & 59 & 0.8944 \\
\hline
\end{tabular}

Statistical analysis between serum levels of C-reactive protein, high-sensitivity form (hsCRP), showed a positive correlation, statistically significant between the level at admission and the number of neutrophils at admission $(\mathrm{p}=0.3169, \mathrm{r}=0.2801)$, respectively a negative correlation, statistically significant between the level at discharge and the level of leukocytes $(\mathrm{p}=$ $0.0253, \mathrm{r}=-0.2911)$, monocytes $(\mathrm{p}=0.0014, \mathrm{r}=$ $-0.4105)$ and neutrophils $(\mathrm{p}=0.0354, \mathrm{r}=-0.2793)$ at discharge. Of the three monitored cytokines, we obtained statistically significant correlations for TNF- $\alpha$. Thus, a positive, statistically significant correlation was identified between lymphocyte values $(\mathrm{p}=$ $0.0007, \mathrm{r}=0.3448)$, monocytes $(\mathrm{p}=0.0056, \mathrm{r}=$ $0.2896)$ and platelets $(\mathrm{p}=0.0335, \mathrm{r}=0.2195)$ at hospitalization with the values of the serum marker TNF- $\alpha$.
Also, a negative correlation was identified, statistically significant between hemoglobin values $(p=0.0034, r=$ $-0.328)$ and hematocrit $(p=0.0004, r=-0.393)$ at discharge with serum marker TNF- $\alpha$ values.

The statistical analysis also included the ratio between neutrophils and lymphocytes (NLR) $(\mathrm{n}=94)$ with an average value of $3,003+/-4.87$, the ratio between neutrophils and platelets (NPR) $(\mathrm{n}=94)$ showing an average value of 0.02 (Std Dev $+/-0.02)$ and the ratio of platelets to lymphocytes (PLR) $(n=94)$ expressing an average value of $127.82+/-101.51$. The obtained results show the following correlations: negative correlation, statistically significant between TNF- $\alpha$ and NLR $(\mathrm{p}=0.0144, \mathrm{r}=-0.2601)$, NPR $(\mathrm{p}=$ $0.0473, r=-0.2051)$, PLR ( $p=0.013, r=-0.2602)$, positive correlation, statistically significant between

TABLE 2. Correlations between NLR, NPR, PLR and inflammatory markers

\begin{tabular}{|c|c|c|c|c|c|c|}
\hline \multirow{2}{*}{$\begin{array}{c}\text { The analyzed } \\
\text { variables }\end{array}$} & \multicolumn{2}{|c|}{ NLR } & \multicolumn{2}{|c|}{ NPR } & \multicolumn{2}{c|}{ PLR } \\
\cline { 2 - 7 } & $n$ & $p$ & $n$ & $p$ & $n$ & $p$ \\
\hline $\mathrm{IL}-1 \mathrm{pg} / \mathrm{ml}$ & 63 & 0.3121 & 68 & 0.6993 & 63 & 0.4517 \\
\hline $\mathrm{IL}-6 \mathrm{pg} / \mathrm{ml}$ & 61 & 0.2093 & 66 & 0.5482 & 62 & 0.8522 \\
\hline $\mathrm{TNF} \mathrm{pg/ml}$ & 88 & $\begin{array}{c}\mathbf{0 . 0 1 4 4} \\
r=-0.2601\end{array}$ & 94 & $\begin{array}{c}\mathbf{0 . 0 4 7 3} \\
r=-0.2051\end{array}$ & 89 & $\begin{array}{c}\mathbf{0 . 0 1 3 8} \\
r=-0.2602\end{array}$ \\
\hline $\begin{array}{c}\mathrm{PCR} \mathrm{mg} / \mathrm{dl}- \\
\text { admission }\end{array}$ & 58 & $\begin{array}{c}\mathbf{0 . 0 1 2 4} \\
r=0.3264\end{array}$ & 59 & $\begin{array}{c}\mathbf{0 . 0 0 7 3} \\
r=0.3460\end{array}$ & 60 & 0.1230 \\
\hline PCR $\mathrm{mg} / \mathrm{dl}-$ discharge & 56 & $\begin{array}{c}\mathbf{0 . 0 1 8 9} \\
r=0.3128\end{array}$ & 59 & 0.2166 & 56 & $\begin{array}{c}\mathbf{0 . 0 0 3 6} \\
r=0.3831\end{array}$ \\
\hline
\end{tabular}


hsCRP at admission and NLR $(\mathrm{p}=0.0124, \mathrm{r}=0.3264)$, NPR $(p=0.0073, r=0.3460)$ and positive correlation, statistically significant between hsCRP at discharge and NLR $(\mathrm{p}=0.0189, \mathrm{r}=0.3128)$, PLR $(\mathrm{p}=0.0036$, $r=0.3031)$. Table 2 shows the values of the correlations made between these reports and the serum inflammatory markers.

\section{DISCUSSIONS}

In most cases, the diagnosis of pneumonia is established based on clinical and radiological criteria. However, there are situations in which further investigations are needed, especially in the overlapping of viral and bacterial infections (13).

The etiology of lower respiratory tract infections differs slightly in the pediatric population from the adult population. In children, viral infections include mainly Human rhinovirus, Human metapneumovirus (HMP) and respiratory syncytial virus (RSV), and among the bacteria Haemophilus influenzae, Staphylococcus aureus and Streptococcus pneumoniae. The treatment also differs between the two populations, as in children there is a very good response to beta-lactams such as Amoxicillin / Ampicillin or cephalosporins, while in adults, due to drug resistance, combined therapies are often needed $(14,15)$.

In our study, 68 patients out of $95(71.27 \%)$ had bacterial pneumonia and needed antibiotic treatment. A recently published meta-analysis monitored numerous studies and literature reviews on empirical antibiotic treatments for pneumonia in infants and children. This article has shown that despite numerous WHO or other international guidelines, antibiotic therapy is still very heterogeneous. Based on the recommendations of the WHO guidelines, pneumonia in children can be very severe, severe or non-severe. Antibiotic therapy is recommended in very severe or severe forms. The first recommended antibiotics are amoxicillin or ampicillin, followed by third-generation cephalosporins (16).

A study carried out on the basis of questionnaires addressed to 120 respondents, most of them pediatricians, showed that there were very large differences in the adherence of prescribers to the recommendations of international guidelines (17).

According to the recommendations of the American Academy of Pediatrics, in each serious case the initial diagnosis will be re-evaluated. In case of atypical forms of pneumonia, atypical or less common etiological agents will be sought. In general, uncomplicated pneumonia will be cured within a maximum of 7 days, under appropriate treatment, including antibiotics. In patients with other comorbidities, especially neuromuscular diseases, pneumonia tends to be severe and often caused by treatment-resistant infectious agents (18). In our study, the average of the hospitalization days was 6.71. Longer hospitalization periods have been reported in patients with comorbidities.

In our study the most used antibiotics were Ceftriaxone, followed by Ampicillin and Clindamycin. Clarithromycin was used in only one patient. Recent studies show the first line in the treatment of pneumonia are $\beta$-lactams. It is preferred that the first antibiotic for hospitalized patients be Penicillin G or Ampicillin. Amoxicillin, whether or not combined with clavulanic acid, is recommended in patients with mild forms. This treatment can be given orally to outpatients. Macrolides are especially recommended in cases of pneumonia with Mycoplasa pneumoniae or Chlamydophila pneumoniae (19-21).

A topic often debated in the literature is related to external risk factors. This category includes domestic or indoor pollution (socio-economic conditions of the family, nutritional status, personal hygiene or one or more smoker between the family members). One of the most important risk factors is the lack of vaccination (immunization), regardless of whether it is bacteria or viruses (including measles) (22). By successfully applying the bivalent vaccine against Haemophilus influenzae type B and Streptococcus pneumoniae, the incidence of respiratory infections in children has been greatly reduced, especially under 5 years old. Basically, immunization has proven to be the most important method of preventing pneumonia. The recommended treatment for mild forms is oral antibiotic therapy and in severe forms it is parenteral administrated antibiotic therapy (23). Our study also included 11 patients considered social cases.

The diagnosis and management of pediatric patients with pneumonia applies to all types of clinical and paraclinical investigations: imaging diagnosis, microbiological diagnosis, laboratory tests - complete blood count and serological markers (procalcitonin - PCT and C-reactive protein). Each of these helps to differentiate viral from bacterial pneumonia, and finally in the indication or not of antibiotic therapy (24). The concentration of procalcitonin (PCT) below $2.5 \mathrm{ng} / \mathrm{ml}$ correlates with mild infections, and high concentrations correlate with severe forms, which require emergency presentation and are associated with complications and prolonged hospitalization. PCT could be used as a biomarker to guide the need for antibiotic treatment (25).

In a multicenter, retrospective cohort study, the prevalence of bacteremia in children with ages between 3 months and 18 years, who were hospitalized 
with pneumonia, was analyzed. Several parameters were analyzed to identify predictors for bacteremia. Thus, ROC curves were performed for the total number of C-reactive protein, ESR and leukocytes. The most important predictors as independent factors associated with bacteremia were leukocytosis, the characteristic radiological appearance and pleural complications, the presentation of the patient in emergency system and the number of days of hospitalization.

According to the authors, as the prevalence of bacteremia was very low, of $2.2 \%$ ( 1 in 400 patients), blood cultures in these patients should be discouraged (26).

In our study we monitored the classic parameters for assessing inflammatory status: complete blood count, body temperature and C- reactive protein at admission and discharge. In order to establish the clinical-biological correlations, we also dosed the modern inflammatory markers, from the blood collected at hospitalization: IL- $1 \beta$, IL- 6 , TNF- $\alpha$.

Numerous recent studies have reported the analysis of biomarkers in various infectious (pneumonia) or non-infectious pathologies (acute myocardial infarction or chronic inflammatory bowel disease). A prospective observational study performed on a group of 66 patients analyzed the predictive value of proadrenomedulin (Pro-ADM) and interleukin 1 beta (IL-1 $\beta$ ) to stage the severity of the disease. These biomarkers were determined from blood samples taken from patients at the time of admission and were analyzed compared to the blood count and C-reactive protein, in parallel with the severity of the disease. Pro-ADM proved to be the best biomarker for staging the severity of the disease, and both biomarkers had increased serum values in patients with complications due to the disease (27).

Another prospective cohort study that included 196 patients with pneumonia, tested 21 cytokines and systemic chemokines. The strongest statistical associations were recorded between the severity of the disease and IL-6, IL-8, IL-13 and IFN- $\gamma$. As in our study, no statistically significant correlations were obtained between the total number of leukocytes and cytokines (28). The relationship between IL-6 (proinflammatory cytokine) and IL-10 (anti-inflammatory cytokine) was also studied to establish the severity of the disease and the patient's evolution, respectively the attempt to establish the bacterial or viral etiology of pneumonia (29).
A prospective study of 322 patients under 5 years old, of which 166 patients were finally selected, analyzed numerous cytokines (IL-1 $\beta$, IL-2, IL-4, IL-5, IL -6, IL-8, IL-10, IL-12, TNF- $\alpha$ and IFN- $\gamma$ ) and chemokines (CCL2, CCL5, CXCL9, CXCL10). Of these, IL-6, IL-8, IL-10, CCL2, and CXCL10 had detectable values. IL-6 had a predictive value for pneumococcal pneumonia (30). Another study included 430 children with severe and non-severe pneumonia where 27 cytokines were tested. Of these, IL- 6 and G-CSF (granulocyte-colony stimulating factor) were correlated with disease severity (31).

The relationship between the values of the elements in the peripheral blood and their predictive value is a widely studied topic today. These indicators are used in chronic inflammatory diseases such as systemic lupus or in metabolic syndrome $(32,33)$. These reports are particularly useful for assessing the response to chemotherapy of different types of cancer (34). They have also been studied in the case of pneumonia, both in children and adults $(35,36)$. In our study we analyzed all three reports: NLR, NPR and PLR and found significantly statistical correlations in pediatric patients with pneumonia, for hsCRP and TNF- $\alpha$.

The main limitations of our study were the relatively small number of patients included in the study $(n=95)$ due to the particularity of the pathology and the performance of the study in only one center. Another limitation was the measurement of biomarkers only from the blood collected at the admission of the patients and the analysis of only a small number of cytokines (IL-1 $\beta$, IL- 6 and TNF- $\alpha$ ), with the lack of analysis of others already reported in the literature (procalcitonin, IL-8, IL-10, IFN- $\gamma$, CCL2, and CXCL10).

\section{CONCLUSIONS}

Pneumonia in children is associated with an inflammatory status of varying degrees depending on the type of pneumonia. Our study showed that the values of neutrophils, lymphocytes, monocytes and platelets, NLR, PLR and TNF- $\alpha$ were significantly increased in pneumonia, correlating with $\mathrm{C}$-reactive protein, highlighting the early inflammatory status in this condition. Early determination of the inflammatory status associated with pneumonia in children is essential to make a diagnosis as soon as possible and prevent complications that may occur. 


\section{REFERENCES}

1. Arbo A, Lovera D, Martínez-Cuellar C. Mortality Predictive Scores for Community-Acquired Pneumonia in Children. Curr Infect Dis Rep. 2019;21(3):10.

2. DeAntonio R, Yarzabal JP, Cruz JP, Schmidt JE, Kleijnen J. Epidemiology of community-acquired pneumonia and implications for vaccination of children living in developing and newly industrialized countries: A systematic literature review. Hum Vaccin Immunother. 2016;12(9):2422-2440.

3. Fritz $C Q$, Edwards KM, Self WH, et al. Prevalence, Risk Factors, and Outcomes of Bacteremic Pneumonia in Children. Pediatrics. 2019;144(1):e20183090.

4. Nathan AM, Teh CSJ, Jabar KA, et al. Bacterial pneumonia and its associated factors in children from a developing country: A prospective cohort study. PLoS One. 2020;15(2):e0228056.

5. Dean P, Florin TA. Factors Associated With Pneumonia Severity in Children: A Systematic Review. J Pediatric Infect Dis Soc. 2018;7(4):323-334.

6. Krawiec M, Kraj G, Peradzynska J, Krauze A, Kulus M. Risk factors for local complications in children with community-acquired pneumonia. Clin Respir J. 2018;12(1):253-261.

7. Jain S, Self WH, Wunderink RG, et al. Community-Acquired Pneumonia Requiring Hospitalization among U.S. Adults. N Engl J Med. 2015;373(5):415-427.

8. Jain S, Williams DJ, Arnold SR, et al. Community-acquired pneumonia requiring hospitalization among U.S. children. $N$ Engl J Med. 2015;372(9):835-845.

9. Shi T, McLean K, Campbell H, Nair H. Aetiological role of common respiratory viruses in acute lower respiratory infections in children under five years: A systematic review and meta-analysis. J Glob Health. 2015;5(1):010408.

10. Slack MPE. The evidence for non-typeable Haemophilus influenzae as a causative agent of childhood pneumonia. Pneumonia (Nathan). 2017;9:9.

11. Principi N, Esposito S. Biomarkers in Pediatric Community-Acquired Pneumonia. Int J Mol Sci. 2017;18(2):447.

12. Nascimento-Carvalho EC, Vasconcellos ÂG, Clarêncio J, et al. Evolution of cytokines/chemokines in cases with community-acquired pneumonia and distinct etiologies. Pediatr Pulmonol. 2020;55(1):169176.

13. Yun KW, Wallihan R, Juergensen A, Mejias A, Ramilo O. CommunityAcquired Pneumonia in Children: Myths and Facts. Am J Perinatol. 2019;36(S 02):S54-S57.

14. Leung AKC, Wong AHC, Hon KL. Community-Acquired Pneumonia in Children. Recent Pat Inflamm Allergy Drug Discov. 2018;12(2):136144.

15. Rider AC, Frazee BW. Community-Acquired Pneumonia. Emerg Med Clin North Am. 2018;36(4):665-683.

16. Mathur S, Fuchs A, Bielicki J, Van Den Anker J, Sharland M. Antibiotic use for community-acquired pneumonia in neonates and children: WHO evidence review. Paediatr Int Child Health. 2018;38(sup1):S66S75.

17. McLaren SH, Mistry RD, Neuman MI, Florin TA, Dayan PS. Guideline Adherence in Diagnostic Testing and Treatment of Community-Acquired Pneumonia in Children [published online ahead of print, $2019 \mathrm{Feb}$ 14]. Pediatr Emerg Care. 2019;10.

18. Messinger Al, Kupfer O, Hurst A, Parker S. Management of Pediatric Community-acquired Bacterial Pneumonia. Pediatr Rev. 2017;38(9):394-409.
19. Nascimento-Carvalho AC, Nascimento-Carvalho CM. Clinical management of community-acquired pneumonia in young children. Expert Opin Pharmacother. 2019;20(4):435-442.

20. Blyth CC, Gerber JS. Macrolides in Children With Community-Acquired Pneumonia: Panacea or Placebo?. J Pediatric Infect Dis Soc. 2018;7(1):71-77.

21. Donà $D$, Luise $D, D a$ Dalt L, Giaquinto $C$. Treatment of CommunityAcquired Pneumonia: Are All Countries Treating Children in the Same Way? A Literature Review. Int J Pediatr. 2017;2017:4239268.

22. Gothankar J, Doke P, Dhumale G, et al. Reported incidence and risk factors of childhood pneumonia in India: a community-based cross-sectional study. BMC Public Health. 2018;18(1):1111.

23. le Roux DM, Zar HJ. Community-acquired pneumonia in children - a changing spectrum of disease. Pediatr Radiol. 2017;47(11):13921398.

24. Zar HJ, Andronikou S, Nicol MP. Advances in the diagnosis of pneumonia in children. BMJ. 2017;358:j2739.

25. Stockmann C, Ampofo K, Killpack J, et al. Procalcitonin Accurately Identifies Hospitalized Children With Low Risk of Bacterial Community-Acquired Pneumonia. J Pediatric Infect Dis Soc. 2018;7(1):46-53.

26. Lipsett SC, Hall M, Ambroggio L, et al. Predictors of Bacteremia in Children Hospitalized With Community-Acquired Pneumonia. Hosp Pediatr. 2019;9(10):770-778.

27. Korkmaz MF, Güzel A, Açıkgöz M, Okuyucu A, Alaçam H. Reliability of Pro-adrenomedullin and Interleukin $1 \beta$ in Predicting Severity of Community-Acquired Pneumonia in Pediatric Patients. Ann Clin Lab Sci. 2018;48(1):81-89.

28. Saghafian-Hedengren S, Mathew JL, Hagel E, et al. Assessment of Cytokine and Chemokine Signatures as Potential Biomarkers of Childhood Community-acquired Pneumonia Severity: A Nested Cohort Study in India. Pediatr Infect Dis J. 2017;36(1):102-108.

29. Conway SR, Doughty LA. Cytokine Help for Diagnosing CommunityAcquired Pneumonia. Pediatr Crit Care Med. 2017;18(4):391-393.

30. Vasconcellos ÂG, Clarêncio J, Andrade D, Cardoso MA, Barral A, Nascimento-Carvalho CM. Systemic cytokines and chemokines on admission of children hospitalized with community-acquired pneumonia. Cytokine. 2018;107:1-8.

31. Haugen J, Chandyo RK, Brokstad KA, et al. Cytokine Concentrations in Plasma from Children with Severe and Non-Severe Community Acquired Pneumonia. PLoS One. 2015;10(9):e0138978.

32. Qin B, Ma N, Tang Q, et al. Neutrophil to lymphocyte ratio (NLR) and platelet to lymphocyte ratio (PLR) were useful markers in assessment of inflammatory response and disease activity in SLE patients. Mod Rheumatol. 2016;26(3):372-376.

33. Liu CC, Ko HJ, Liu WS, et al. Neutrophil-to-lymphocyte ratio as a predictive marker of metabolic syndrome. Medicine (Baltimore). 2019;98(43):e17537.

34. Graziano V, Grassadonia A, lezzi L, et al. Combination of peripheral neutrophil-to-lymphocyte ratio and platelet-to-lymphocyte ratio is predictive of pathological complete response after neoadjuvant chemotherapy in breast cancer patients. Breast. 2019;44:33-38.

35. Huang Y, Liu A, Liang L, et al. Diagnostic value of blood parameters for community-acquired pneumonia. Int Immunopharmacol. 2018;64:10-15.

36. Cataudella E, Giraffa CM, Di Marca S, et al. Neutrophil-To-Lymphocyte Ratio: An Emerging Marker Predicting Prognosis in Elderly Adults with Community-Acquired Pneumonia. J Am Geriatr Soc. 2017; 65(8):1796-1801. 